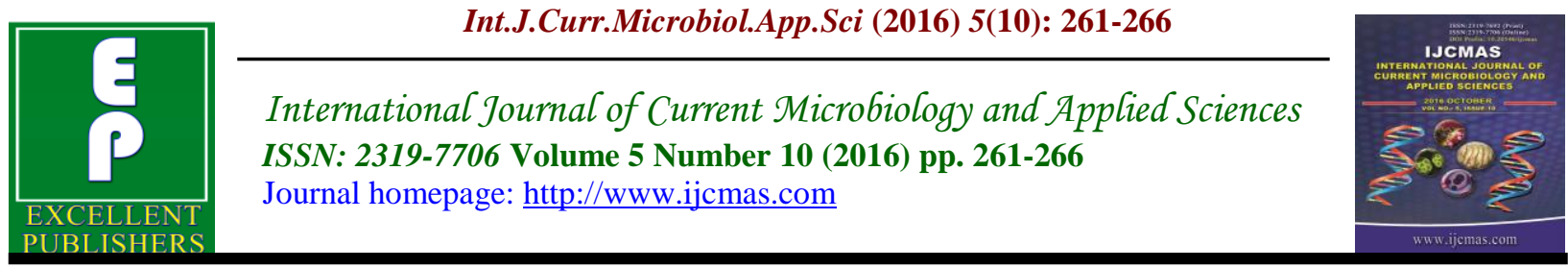

Original Research Article

http://dx.doi.org/10.20546/ijcmas.2016.510.029

\title{
Gold Nanoparticles for Photothermal Therapy of Cancerous Cells in vitro
}

\author{
Sumaiah I. Hussein ${ }^{1 *}$, Ali S. Sultan ${ }^{2}$ and Nahi Y. Yaseen \\ ${ }^{1}$ Department of Basic Science, College of Dentistry, Baghdad University, Iraq \\ ${ }^{2}$ Department of Biology, College of Science, AL-Mustansiriya University, Iraq \\ *Corresponding author
}

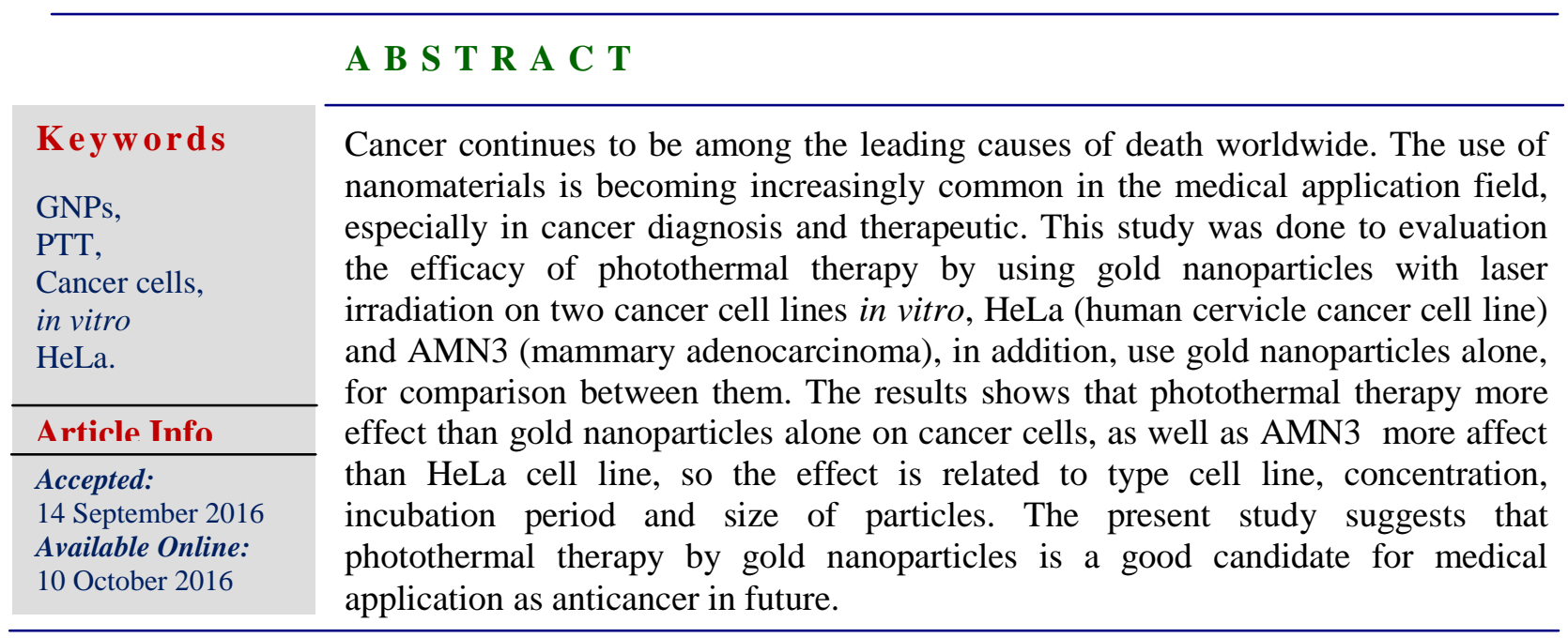

\section{Introduction}

Nanotechnology offers unique approach of controlling variety of biological and medical processes at nanoscale, Recent applications of nanotechnology involve use of nanoparticles for the treatment of variety of disease like cancer, diabetes, HIV vaccine. Conventional cancer therapy involves a cytotoxic agent that often shows the harmful side effects (Butle and Baheti, 2011).

Nanoparticle is a particle with the dimension of $100 \mathrm{~nm}$ or less, size-dependent properties of physics at the nanoscale typically develop.

Gold nanoparticles (GNPs) one of these material which possess optical properties such as strong absorption scattering in the visible -near infrared region or green light depending on their size and shape, based on these optical properties, GNPs have been used in various types of biomedical application including photothermal therapy, biosensering, Drug delivery and gene therapy (Jain et al., 2008). The use of GNPs in cancer diagnosis and therapy has been widely investigated (EL-Sayed et al., 2006), because these particles are known to have excellent surface plasmon resonance and strong biocompatibility, such as antioxidant, nontoxin, chemical stability and high affinities to biomolecules (Huisman et al., 2005). 
Photothermal therapy (PTT) by using GNPs have been used to destroy target cancer cells with minimum damage to normal tissue, in addition, GNPs absorb light strongly and convert photon energy into heat quickly with efficiently, Besides scattering, GNPs also absorb light million of time more than organic dye molecule, almost $100 \%$ of the absorbed light is converted to heat (Iancu, 2013). Chen et al., (2015) suggested that Photothermal therapy consider as optimal treatment approach because the malignant cells required only one third of the energy require for control or non-malignant cells.

\section{Materials and Methods}

Gold nanoparticles (GNPs) prepared in Sigma Aldrich Company with 10nm, spherical shape and at concentration $6 \times$ 1012 particles $/ \mathrm{ml}$.

\section{Cell culture}

Human cervical cancer (HeLa) and Mammary adenocarcinoma (AMN3) cell lines were purchased from tissue culture unit/ Iraqi Centre for Cancer and Medical Genetics Research (ICCMGR), the cells were cultured in $50 \mathrm{~cm} 2$ tissue culture flasks under humidified 5\% $\mathrm{CO} 2$ atmosphere at $37^{\circ} \mathrm{C}$ in RPMI-1640 medium (Sigma chemical) with $10 \%$ fetal bovine serum (FBS), and peniciline-streptomycin during the experiment.

\section{Cytotoxicity assay}

From stock solution of GNPs and by using free serum media, five concentrations (dilution) of GNPs $(9 \times 1011,3 \times 1011$, $1 \times 1011,36 \times 108$ and $12 \times 108)$ particles $/ \mathrm{ml}$ were prepared, then, the cell culture in microtiter plate (96 well) and during log phase of growth were exposed to five concentration of GNPs with four replicate at
$200 \mu 1$ for each concentration, while the control well were exposed to only free serum medium, the plats were incubated for two incubation period 24 and 48 hours.

After incubation period, the cells staining by MTT stain and read by micro-ELISA reader at wavelength on $550 \mathrm{~nm}$, then, the inhibition rate was calculated as follows:

$\mathrm{IR} \%=\mathrm{A}-\mathrm{B} / \mathrm{A} \times 100$

$\mathrm{IR}=$ inhibitor rate, $\mathrm{A}=$ the optical density of control, $\mathrm{B}=$ the optical density of treatment.

\section{Irriadation procedure}

After added GNPs to wells as previous procedure, the following parameters were used in Irradiation procedure in vitro via Low Level Laser (LLL), continuous laser (green light) $532 \mathrm{~nm}$, output power is equal to $112 \mathrm{~mW}$, the power of laser was measured using power meter, beam diameter at laser aperture is equal to $4 \mathrm{~mm}$. The exposure time for 4 min through $24 \mathrm{hrs}, 8 \mathrm{~min}$ through 48 (4 min for each $24 \mathrm{hrs}$ ), Power density (0.083 $\mathrm{W} / \mathrm{cm} 2)$.

\section{Results and Discussion}

Comparison between effect of gold nanoparticles alone and photothermal therapy

Figure (1) and (2) illustrates the comparison between treatment of GNPs alone and treatment of GNPs plus laser radiation (photothermal therapy) for $4 \mathrm{~min}$ for each day in HeLa cell line. The figure shows the treatment with PTT has obtained inhibition rate more than treatment with GNPs alone in all concentrations and in two period of exposure with highly significant at level $(\mathrm{P}<0.01)$. After the cancer cells were exposed to GNPs for $24 \mathrm{hrs}$, the first 
concentration has higher inhibition rate, while when it exposed to PTT for 4min through $24 \mathrm{hrs}$, the third concentration has higher inhibition rate.

After cancer cells exposed to GNPs for 48hrs, inhibition rate was occurred in third concentration, but in PTT for 4min (twice through 48hrs), the last concentration has higher inhibition rate.

The same the thing was noticed for AMN3 cell line, but the first concentration has higher inhibition rate after exposed to GNPs and PTT (4min) through 24hrs, as well as through 48hrs, the first concentration has higher inhibition rate when it exposed to GNPs, so the first and third concentration has higher inhibition rate after PTT.

In this study, the photothermal therapy was applied via employed spherical gold nanoparticles (GNSPs) was with continuous wave $(\mathrm{CW})$ visible laser $(532 \mathrm{~nm})$ due to the surface plasmonic resonance (SPR) absorption in the visible region of (GNSPs) (Myroshnychenko et al., 2008; Honda et al., 2011).

Photothermal therapy is very desirable due to the sensitivity of cancer cells to this type of treatment, in addition, minimum damage to normal cells and surrounding tissue (ALSayed et al., 2006).

Radiation with diode laser started after one hour from added GNPs to cancer cells according to Connor et al., (2005) which proved that maximum GNPs uptake was occurred after one hour from exposure. So the use of $\mathrm{CW}$ laser is advantageous for effective heat accumulation in order to induce mild cell death in a larger area. In addition, using CW laser for treatment is more time consuming (minutes than pulse laser (single pulse time). Hence, the PTT by $\mathrm{CW}$ lasers has been employed in selective cancer cells death (El-Sayed et al., 2006).

The study by Song et al., (2012) revealed that GNPs with different size rapidly internalized in to HeLa and human breast cancer, by TEM images, GNPs were mainly located at the cytoplasm, mitochondria and even in nucleus, after that the laser can be specifically tuned to the SPR frequency of nanoparticles, varying with their size, shape and composition (Lal et al., 2008; Lodice et al., 2016).

Fig.1 Shows comparison between the effect of GNPs and photothermal therapy on HeLa cell line at $24 \mathrm{hrs}$

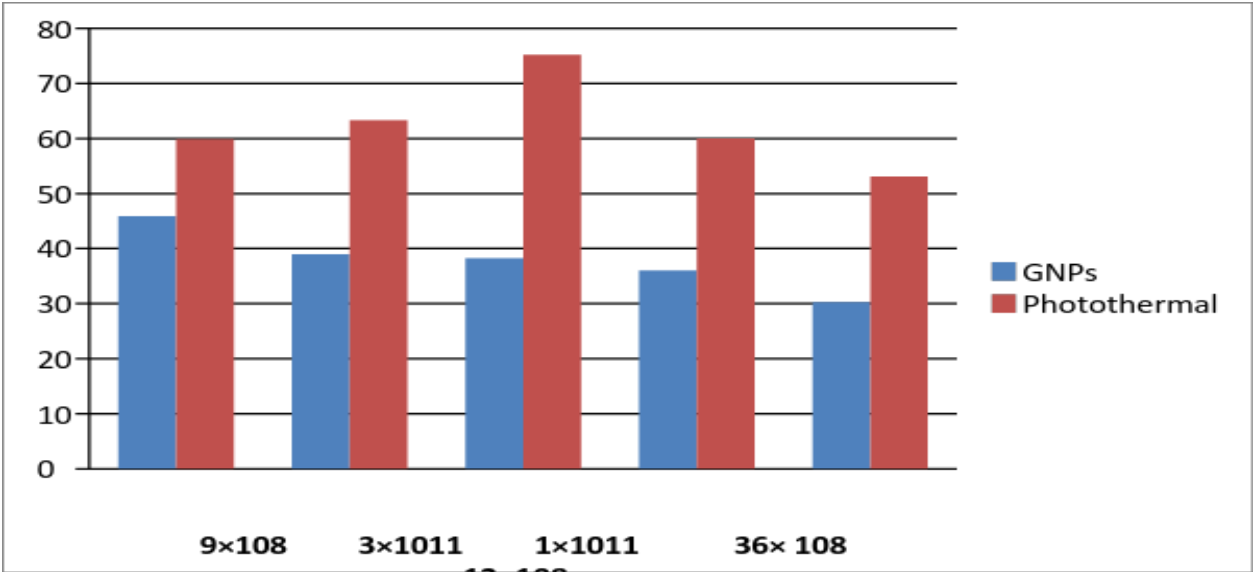


Fig.2 Shows comparison between the effect of GNPs and photothermal therapy on HeLa cell line at $48 \mathrm{hrs}$

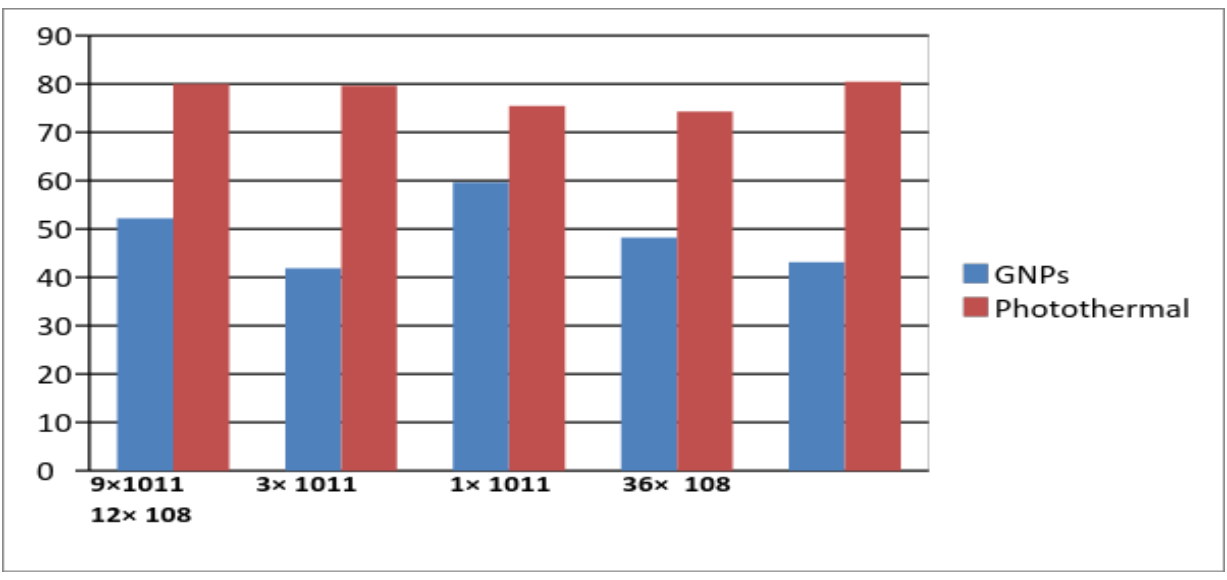

Fig.3 Shows comparison between effect of GNPs and photothermal therapy on AMN3 cell line at $24 \mathrm{hrs}$

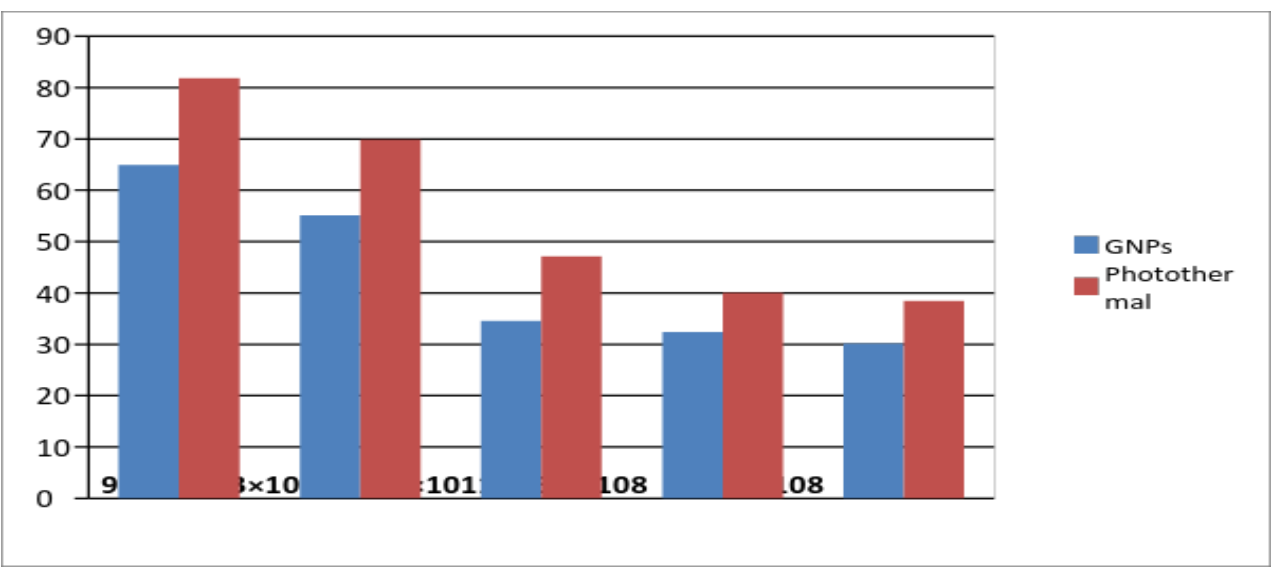

Fig.4 Shows comparison between the effect of GNPs and photothermal therapy on AMN3 cell line at 48hrs.

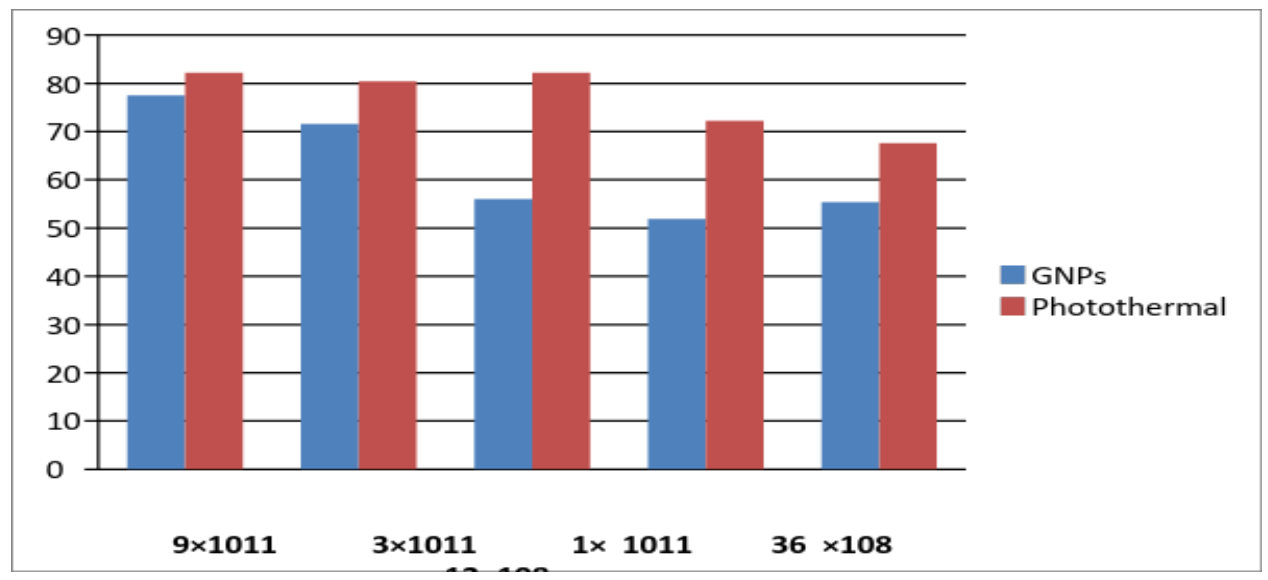


In photothermal therapy experiment, the action of cytotoxicity is converted to higher rate of growth inhibition especially in 48hrs, because the GNPs can effective in PTT by generate vapor nanobubbles when the particles are locally overheated with laser, due to the evaporation offer very thin volume of the surrounding medium (Lukianova-Hleb et al., 2011), while Chen and coworker (2015) suggested that cancer cells killing with GNPs required about only one third of energy required for the control group cancer cell lines without treatment by GNPs.

The study of photothermal therapy by Huang and others (2006) found that cancer cells with higher concentration of GNPs required a lower laser radiation power to achieve the temperature rise for cell destruction, this explain why the inhibition rate was increased in photothermal therapy with higher concentration of GNPs in this study, while the obtained results by Song and colleagues (2012) proved that even the lower doses of GNPs can still enhance killing effect using radiation.

The same thing in the report of Huang et al., (2012) when they found severe increase in cell death after laser radiated in the presence of GNPs than treated with only gold or laser alone.

However, the researcher Servies (2016) suggested that large cluster of GNPs lead to rise of temperature, then to vaporize adjacent water molecules which creating tiny bubbles that quickly expand and burst, ripping apart of cancer cells but not the normal cells. Therefore, the present study suggest that PTT by using GNPs has potential future in medical application, and strong candidate for cancer treatment.

\section{References}

Butle, S.R., Baheti, P.R. 2011. Role of goldnanoparticles in the detection and treatment of cancer. Int. J. Pharma. Sci. Rev. Res., 10: 54-59.

Chen, C.H., Wu, Y.J. and Chen, J.J. 2015. Gold nanotheranostics: photothermal therapy and imaging of mucin 7 conjugated antibody nanoparticles for urothelial cancer. BioMed. Res. Int., 8.

Connor, E.E., Mwamuka, J., Gole, A., Murphy, C.J., Wyatt, M.D. 2005. Gold nanoparticles are taken up by human cells but do not cause acute cytotoxicity. Small, 1: 325327.

EL-Sayed, I.V., Huang, X. and EL-Sayed, M.A. 2006. Selective laser photothermal therapy of epithelial carcinoma using anti-EGFRantibody conjugated gold nanoparticles. Cancer Lett., 239: 129-135.

Honda, M., Saito, Y., Smith, N. I., Fujita, K. and Kawata, S. 2011. Nanoscale heating of laser irradiated single gold nanoparticles in liquid. Optics Express, 19: 12375-12383.

Huang, K., Ma, H., Liu, J., Huo, S., Kumar, A., We, T., Zhang, X., Jin, S., Gan, Y., Wang, P., He, S. and Liang, X. 2012. Size dependent localization and penetration of ultrasmall gold nanoparticles in cancer cells, multicellular spheroids, and tumor in vivo. ACS Nano, 6: 4483-4493.

Huang, X., Jain, P.K., EL-Sayed, H.I. and EL-Sayed, M.A. 2006. Determinaton of the minimum temperature required for selective photothermal destruction of cancer cells with the use of immunotargeted gold nanoparticles. Photochem. Photobio., 82: 412-417.

Huisman, H.J., Futterer, J.J., Van Lin, E.N. et al. 2005. Prostate cancer, precision of integrating functional MR imaging with radiation therapy treatment by 
using fiducial gold markers. Radiol., 236(1): 311-317.

Iancu, C. 2013. Photothermal therapy of human cancers PTT using gold nanoparticles. Biotechnol. Mol. Bio Nanomed., 1: 53-60.

Jain, P.K., Huang, X., EL-Sayed, I.H. and EL-Sayed, M.A. 2008. Nobel metal on the nanoscale: optical and photothermal properties and some applications in imaging, sensing, biology and medicine. Accounts of Chem. Res., 41(12): 1578-1586.

Lal, S., Clare, S.E. and Halas, N.J. 2008. Nanoshell-enable photothermal cancer therapy:impending clinical impaxt. ACC Chem. Res., 41: 1842-1851.

Lodice, C., Cervadoro, A., Palange, A., Key, J., Aryal, S., Ramirez, M.R., Mattu, C., Cianluca, G., O, Neill, B.E. and Decuzzi, P. 2016. Enhancing photothermal cancer therapy by clustering gold nanoparticles into spherical polymeric nanoconstructs. Optics Laser Engineer, 76: 74-81.

Lukianova-Hleb, E.Y., Oginsky, A.O. and Samaniego, A.P. 2011. Tunable plasmonic nanoprobes for theranostics of prostate cancer. Theranostics, 1: 317.

Myroshnychenko, V., Rodriguez-Fernandez, J., Pastoriza-Santos, I., Funston, A. M., Novo, C. and Mulvaney, P. 2008. Modelling the optical response of gold nanoparticles. Chem. Soc. Rev., 37: 1792-1805.

Service, R.F. 2016. Exploding nanobubbles can kill cancer cells. Sci., 352: issue 6281.

Song, K., Xu, P., Meng, Y., Geng, F., Li, J., Li, Z., Xing, J., Chen, J. and Kong, B. 2012. Smart gold nanoparticles enhance killing effect on cancer cells. Int. J. Oncol., 42: 597-608.

\section{How to cite this article:}

Sumaiah I. Hussein, Ali S. Sultan and Nahi Y. Yaseen. 2016. Gold Nanoparticles for Photothermal Therapy of Cancerous Cells in vitro. Int.J.Curr.Microbiol.App.Sci. 5(10): 261266. doi: http://dx.doi.org/10.20546/ijcmas.2016.510.029 